Emilio Andrea Maugeri^, Jörg Neuhausen, Ryszard Misiak, Robert Eichler, Rugard Dressler, David Piguet, Alexander Vögele and Dorothea Schumann

\title{
Adsorption of volatile polonium species on metals in various gas atmospheres: Part II - Adsorption of volatile polonium on platinum, silver and palladium
}

DOI 10.1515/ract-2016-2575

Received January 12, 2016; accepted May 13, 2016; published online July 2, 2016

\begin{abstract}
This work presents the results obtained from studying the interaction between polonium compounds formed in different atmospheres and platinum, palladium and silver surfaces obtained by thermochromatography. These results are of crucial importance for the design of cover gas filter systems for lead-bismuth eutectic (LBE)-based nuclear facilities such as accelerator driven systems (ADS). The results obtained from studying the interaction of polonium and platinum under inert atmosphere and reducing atmospheres with and without addition of moisture show that polonium is deposited at temperatures between 993 and $1221 \mathrm{~K}$, with adsorption enthalpies ranging from -235 to $-291 \mathrm{~kJ} \mathrm{~mol}^{-1}$, indicating a very strong adsorption of the polonium species present on platinum surfaces. The interaction between polonium and silver was investigated using purified inert, reducing and oxidizing carrier gases. Results show a deposition temperature between 867 and $990 \mathrm{~K}$, with adsorption enthalpies ranging from -205 to $-234 \mathrm{~kJ}$ $\mathrm{mol}^{-1}$. The interaction of polonium and palladium was studied in purified helium and purified hydrogen. For both conditions a deposition temperature of $1221 \mathrm{~K}$ was observed corresponding to an adsorption enthalpy of $-340 \mathrm{~kJ} \mathrm{~mol}^{-1}$. No highly volatile polonium species was formed at any of the applied experimental conditions.
\end{abstract}

Keywords: Polonium, thermochromatography, ADS, MYRRHA.

\footnotetext{
*Corresponding author: Emilio Andrea Maugeri, Laboratory for Radiochemistry, Paul Scherrer Institut, Villigen PSI, CH-5232 Villigen, Switzerland, E-mail: emilio-andrea.maugeri@psi.ch Jörg Neuhausen, Rugard Dressler, David Piguet, Alexander Vögele and Dorothea Schumann: Laboratory for Radiochemistry, Paul Scherrer Institut, Villigen PSI, CH-5232 Villigen, Switzerland Ryszard Misiak: The Henryk Niewodniczański Institute of Nuclear Physics, Radzikowskiego 152, 31-342 Kraków, Poland Robert Eichler: Laboratory for Radiochemistry, Paul Scherrer Institut, Villigen PSI, CH-5232 Villigen, Switzerland; and Department for Chemistry and Biochemistry, University of Berne, $\mathrm{CH}-3012$ Bern, Switzerland
}

\section{Introduction}

Several years ago the concept of "partitioning and transmutation" was proposed to reduce the long-term radiotoxicity of the spent nuclear fuel and high level waste, one of the main technological challenges and public concerns of the nuclear energy industry.

According to this concept the long-lived minor actinides (MAs) neptunium, americium, and curium and some long-lived fission products (LLFPs), e. g. ${ }^{129} \mathrm{I},{ }^{137} \mathrm{Cs}$, ${ }^{135} \mathrm{Cs}$, ${ }^{79} \mathrm{Se},{ }^{126} \mathrm{Sn}$ and ${ }^{99} \mathrm{Tc}$, should be separated from the spent nuclear fuel and subsequently transmuted in radionuclides with shorter half-lives [1]. It was estimated that the complete or the partial removal of the MAs from the spent nuclear fuel would decrease its intrinsic radiotoxicity by a factor of 100-1000 [2]. High efficiencies of MAs transmutation are obtained using fast neutrons, which can be supplied by nuclear fast reactor or accelerator driven system (ADS) facilities.

The use of fast neutrons for burning MAs in an ADS was first described by Rubbia [3]. An ADS consists of three main parts: a high intensity proton accelerator, a target of heavy nuclei and a subcritical core where MAs and LLFPs are loaded. The high-energy beam of protons is shot at the target of a heavy nuclei, producing neutrons by spallation. Those neutrons are used to drive the nuclear fission chain reaction in the subcritical core and, at the same time, to transmute both MAs and LLFPs. Different high-Z materials can be used as targets, e.g. tungsten, tantalum, depleted uranium, thorium, zirconium, lead, lead-bismuth, mercury. Among those target materials, lead-bismuth eutectic (LBE) which offers different advantages such as chemical stability, can be operated at low temperature due to its low melting temperature (396 K), high boiling point, low reactivity with water and air and a good neutronic performance [4]. Thanks to those properties LBE can be used also as a reactor coolant.

On the other hand, LBE when used both as target material and coolant, is subjected to high fluxes of high energetic protons and neutrons, which cause the formation of ${ }^{208-210} \mathrm{Po}$ isotopes. 
Polonium isotopes are highly radiotoxic. Additionally, they may form volatile species which lead to their release into the cover gas of the ADS system. This represents the main radiological hazard in both operation conditions and accident scenarios. Thus, an adequate gas phase filtering system must be implemented.

In this work the use of noble metals such as platinum, silver and palladium as potential adsorption materials for volatile polonium species is evaluated. The thermochromatography method was used to assess the affinity of monoatomic polonium and of other volatile polonium species to those metals. This method was already successfully used to determine the adsorption enthalpies for polonium on nickel and palladium surfaces [5], on titanium and on copper [6].

The adsorption enthalpies of polonium on platinum, silver, and palladium have been predicted using two different methods: density functional theory (DFT) [7] and the semi-empirical MIEDEMA model described [8].

According to the DFT calculations, the adsorption enthalpy of polonium on platinum surfaces ranges between -289 and $-371 \mathrm{~kJ} \mathrm{~mol}^{-1}$, while according to the MIEDEMA model it is $-260 \mathrm{~kJ} \mathrm{~mol}^{-1}$.

The adsorption enthalpy of polonium on silver surfaces predicted by DFT ranges between -203 and $-244 \mathrm{~kJ}$ $\mathrm{mol}^{-1}$, in fair agreement with the value calculated with the MIEDEMA model, i. e. $-219 \mathrm{~kJ} \mathrm{~mol}^{-1}$.

An adsorption enthalpy of polonium on palladium surfaces between -298 and $-368 \mathrm{~kJ} \mathrm{~mol}^{-1}$ was calculated with the DFT method. The corresponding value obtained using the MIEDEMA model is $-333 \mathrm{~kJ} \mathrm{~mol}^{-1}$.

\section{Experimental}

\subsection{Sample preparation}

${ }^{206}$ Po was used for thermochromatographic investigations because of its easily detectable $\gamma$-ray emission and the suitable half-life of 8.8 days. The isotope ${ }^{206} \mathrm{Po}$ was obtained by irradiation of bismuth metal disks with a $40 \mathrm{MeV}$ proton beam of up to $3 \mu \mathrm{A}$ current at ZAG Karlsruhe. Each irradiated disk was cut into small fragments containing between 5 and $20 \mathrm{kBq}$ of ${ }^{206} \mathrm{Po}$ suitable for one thermochromatography experiment. Polonium was separated from the bismuth matrix and deposited on gold foils before starting the thermochromatography experiments by thermal release using a setup described [9]. Polonium deposited on the gold foils constitutes the starting materials for thermochromatography experiments. The polonium-bismuth separation is not complete. Because of the large excess of bismuth in the irradiated disks, the starting material is still a dilute solution of polonium in bismuth, with a mole fraction of polonium in the range of $1.4-5.6 \times 10^{-6}$. This supports the idea that polonium could evaporate as bismuth polonide rather than monoatomic polonium if the bismuth-polonide molecules are reasonably stable.

\subsection{Thermochromatography}

The thermochromatography method is based on the adsorption-desorption equilibrium between trace amounts [10] of volatile species and a stationary phase at zero surface coverage as a function of the temperature [11]. The experimental result of a thermochromatography experiment consists of a thermochromatogram in which the surface concentration of the adsorbed species is plotted as a function of either its position in the column or the deposition temperature. Standard adsorption enthalpies, $\Delta H_{\text {ads }}^{0}$, of the deposited species on the material constituting the stationary phase were calculated using a Monte Carlo code developed for the simulation of gas thermochromatography experiments [11].

The thermochromatography setup used for investigating the formation and deposition of polonium compounds on noble metals in different atmospheres is described [9]. The stationary phase consisted of a fused silica tube lined inside with foils of platinum, silver and palladium (Goodfellow, thickness $0.020 \pm 0.003 \mathrm{~mm}$, purity $99.9 \%$ ), respectively. The setup, described in detail [9], was equipped with a carrier gas supply allowing for the use of different atmospheres, namely inert, reducing, oxidizing, moist, and dry. The inert $(\mathrm{He})$ and reducing $\left(\mathrm{H}_{2}\right)$ carrier gases were passed through a purification system consisting of a $\mathrm{P}_{2} \mathrm{O}_{5}$ cartridge and a Ta getter heated to $1273 \mathrm{~K}$ to remove moisture and oxygen. Oxygen used as carrier gas was only dried by passing through the $\mathrm{P}_{2} \mathrm{O}_{5}$ cartridge. When using moisturized gases, the carrier gas was passed through a water reservoir at room temperature, bypassing the purification system.

In some experiments, a dew point sensor was added at the outlet of the setup to measure water concentration in the outflowing carrier gas.

Each experiment was performed according to the procedure described [9]. Before each experiment, the noble metal column was heated up to $1273 \mathrm{~K}$ under a constant flow of hydrogen for $120 \mathrm{~min}$ to remove impurities from its surface. Then the noble metal column was flushed with pure $\mathrm{He}$ at the same temperature for $30 \mathrm{~min}$, to remove 
the hydrogen adsorbed on the surface or dissolved into its bulk. The column was finally cooled down to room temperature under a helium flow.

This procedure was used for obtaining oxide-free metal surfaces in the columns before each thermochromatography experiment. In an inert and reducing atmosphere, no oxidation will occur during the experiment. However, when using oxidizing carrier gases, oxidation of the metal surfaces has to be considered. The oxides of the metals used as column materials in this work show very different stabilities. The situation is most complex for platinum oxide. In fact, different ranges of decomposition temperatures are given in the literature for $\mathrm{PtO}_{2}$ in 1 bar oxygen atmosphere, e. g. a range from 550 to $750 \mathrm{~K}$ is given [12], while a later report states a range from 553 to $1023 \mathrm{~K}$ [13]. In another report on the interaction of Pt(111) surfaces with oxygen [14] it is stated that in the temperature range between 170 and $700 \mathrm{~K}$ atomic oxygen is chemisorbed on the Pt-surface, while at temperatures in the range of $800-1100 \mathrm{~K}$ the platinum surface is oxidized, with the oxygen atoms located in subsurface positions. We did not observe any visual change in the surfaces of the platinum columns after experiments performed in oxygen. However, the complex behaviour described above indicates that oxygen adsorption and/or surface oxidation could influence polonium adsorption on platinum in oxygen carrier gas. Silver oxide decomposes at $433 \mathrm{~K}$ in oxygen at a pressure of 1 bar [15]. Finally, palladium oxide dissociates into the elements only above about $1173 \mathrm{~K}$ in oxygen at a pressure of 1 bar $[15,16]$. As a consequence, we expect silver to prevail as a metallic surface at the deposition temperatures observed within this study even under oxidizing conditions. Indeed, palladium shows severe oxidation when oxygen is used as carrier gas.

\section{Results}

\subsection{Thermochromatography of polonium on platinum}

The experimental conditions and the results in terms of deposition temperatures and adsorption enthalpies of all polonium thermochromatography experiments performed in platinum columns are compiled in Table 1.

Figure 1 shows the results of Exp. Pt I and Exp. Pt II, where thermochromatograms of ${ }^{206}$ Po were obtained using purified helium as a carrier gas. The thermochromatogram of Exp. Pt I (a) shows a single sharp peak, centered at $1026 \mathrm{~K}$. Using the Monte Carlo simulation method for gas chromatographic transport [11], the adsorption enthalpy of the deposited species on platinum was calculated as $-246 \pm 7 \mathrm{~kJ} \mathrm{~mol}^{-1}$ assuming an unchanged stable chemical state of the species. Exp. Pt II was performed in the same conditions of Exp. Pt I, i. e. purified helium. The obtained deposition pattern (b) shows a main peak centered at $1058 \mathrm{~K}$ and a small shoulder at $1172 \mathrm{~K}$. Using the Monte Carlo simulation, adsorption enthalpies of $-253 \pm 7$ and $-277 \pm 7 \mathrm{~kJ} \mathrm{~mol}^{-1}$ were determined assuming two chemical species deposited independently. An interconnection of both peaks over a superimposed transport reaction or a surface decomposition reaction of the species deposited at the higher temperature cannot be ruled out.

A series of six thermochromatographic experiments were performed in purified $\mathrm{H}_{2}$, Exp. Pt III-VIII.

Although Exp. Pt III, IV and V were performed using the same setup and nominally the same conditions, the resulting thermochromatograms, presented in Figure $2 \mathrm{a}, \mathrm{b}$ and $\mathrm{c}$, respectively, are different. While the

Table 1: Experimental conditions and results of the ${ }^{206} \mathrm{Po}$ investigations on platinum surfaces.

\begin{tabular}{|c|c|c|c|c|c|}
\hline $\begin{array}{l}\text { Sample } \\
\text { number }\end{array}$ & Carrier gas & $\begin{array}{l}\mathrm{H}_{2} \mathrm{O} \text { content measured at outlet } \\
\text { at end of experiment/ppm }\end{array}$ & $\begin{array}{l}{ }^{\mathrm{a}} \mathbf{T}_{\text {dep }}{ }^{206} \mathrm{Po} / \mathrm{K} \\
\text { (uncertainty }= \pm 30 \mathrm{~K} \text { ) }\end{array}$ & $\begin{array}{r}\Delta H_{\text {ads }} / \mathrm{kJ} \mathrm{mol}^{-1} \\
\text { (uncertainty }= \pm 7 \mathrm{~kJ} \mathrm{~mol}^{-1} \text { ) }\end{array}$ & $\begin{array}{l}\text { Chemical } \\
\text { species }\end{array}$ \\
\hline Exp. Pt I & \multirow[t]{2}{*}{ Purified He } & Not measured & 1026 & -246 & \\
\hline Exp. Pt II & & Not measured & 1172,1058 & $-277,-253$ & \\
\hline Exp. Pt III & \multirow[t]{6}{*}{ Purified $\mathrm{H}_{2}$} & Not measured & 993 (shoulder at 1090) & -256 & \\
\hline Exp. Pt IV & & Not measured & 1090 & -277 & \\
\hline Exp. Pt V & & Not measured & 1172 & -277 & \\
\hline Exp. Pt VI & & 350 & 1190 & -282 & \\
\hline Exp. Pt VII & & 500 & 1145 & -272.5 & \\
\hline Exp. Pt VIII & & 1277 & 1172 & -277 & \\
\hline Exp. Pt IX & Dry $\mathrm{O}_{2}$ & Not measured & 993 & -236 & $\mathrm{PoO}_{2}$ \\
\hline Exp. Pt X & Moist $\mathrm{He}$ & $\geq 23,000$ & 1221 & -291 & \\
\hline Exp. Pt XI & Moist $\mathrm{H}_{2}$ & $\geq 23,000$ & 1212 & -285.5 & \\
\hline
\end{tabular}

aThe deposition temperature, $\mathrm{T}_{\mathrm{dep}}$, is considered as the temperature corresponding to maximum in the polonium deposition pattern. Considering the uncertainties of temperature and position measurement, its uncertainty is estimated to be $\pm 30 \mathrm{~K}$. 


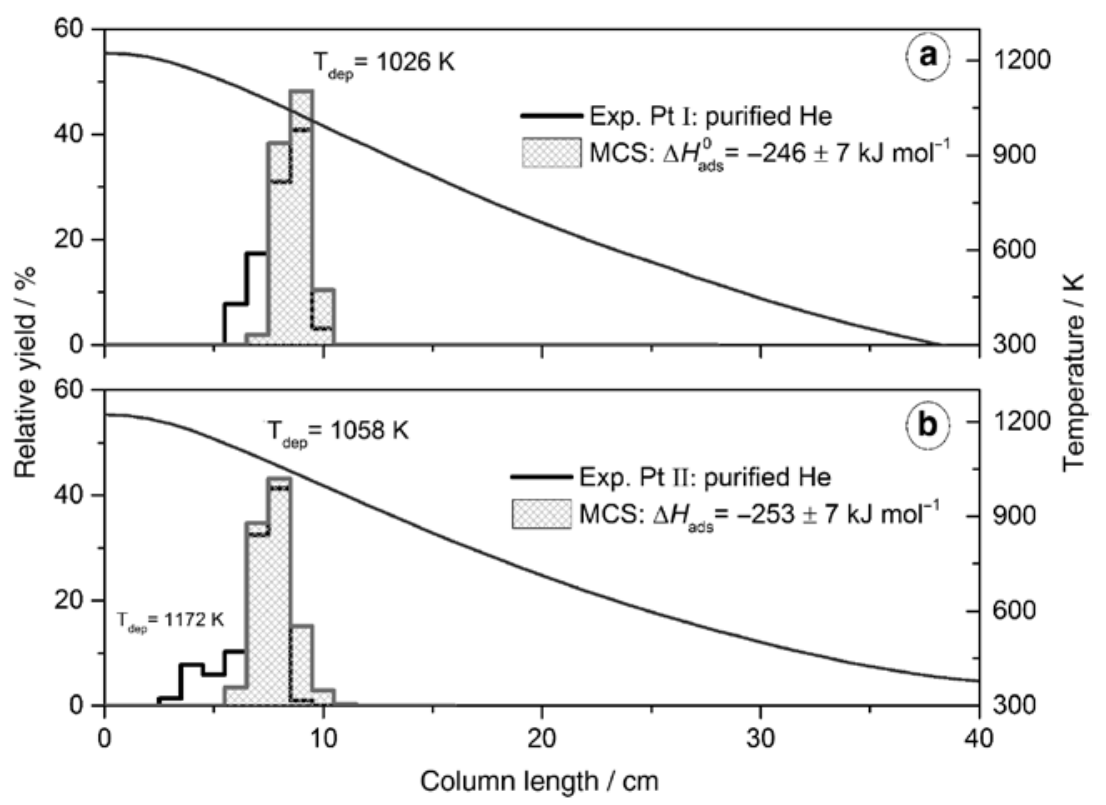

Figure 1: Thermochromatograms of ${ }^{206} \mathrm{Po}$ on platinum using purified He as carrier gas. The black bars show the experimentally determined deposition patterns. The light gray cross-hatched bars represent the corresponding Monte Carlo simulations.

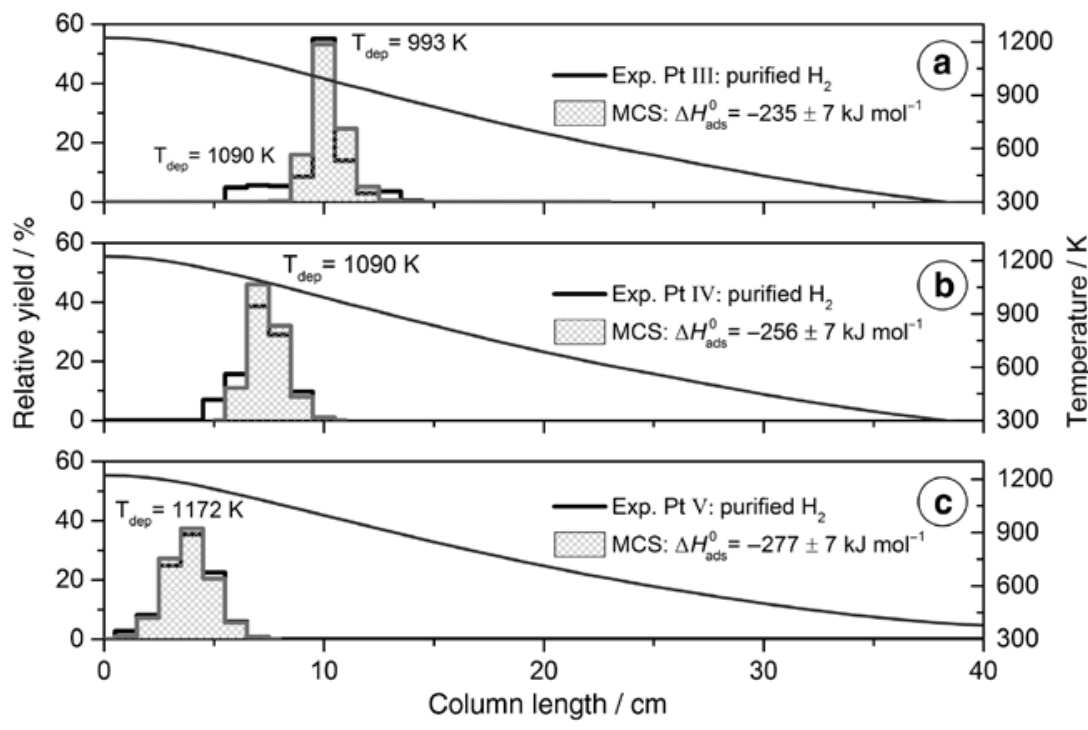

Figure 2: Thermochromatograms of ${ }^{206} \mathrm{Po}$ on platinum using purified $\mathrm{H}_{2}$ as carrier gas. The black bars show the experimentally determined deposition patterns. The light gray cross-hatched bars represent the corresponding Monte Carlo simulations.

thermochromatograms of Exp. Pt IV and V are characterized by a single peak at 1090 and 1172, respectively, the thermochromatogram of Exp. Pt III presents a main peak at $993 \mathrm{~K}$ and a shoulder at $1090 \mathrm{~K}$.

A possible cause of the different results obtained might be the presence of different amounts of water in the carrier gas used. As a consequence, three additional experiments were carried out using the same thermochromatography setup with the addition of a dew point sensor at the outlet of the setup to measure the water concentration in the outflowing carrier gas during the thermochromatography experiments. The obtained values of water concentrations, ranging from 350 to $1277 \mathrm{ppm}$, are reported in Table 1. Figure 3 shows the obtained thermochromatograms, characterized by a single peak between 1190 and $1145 \mathrm{~K}$.

Exp. Pt IX was performed using dry oxygen as a carrier gas. The obtained thermochromatogram, Figure 4a, 


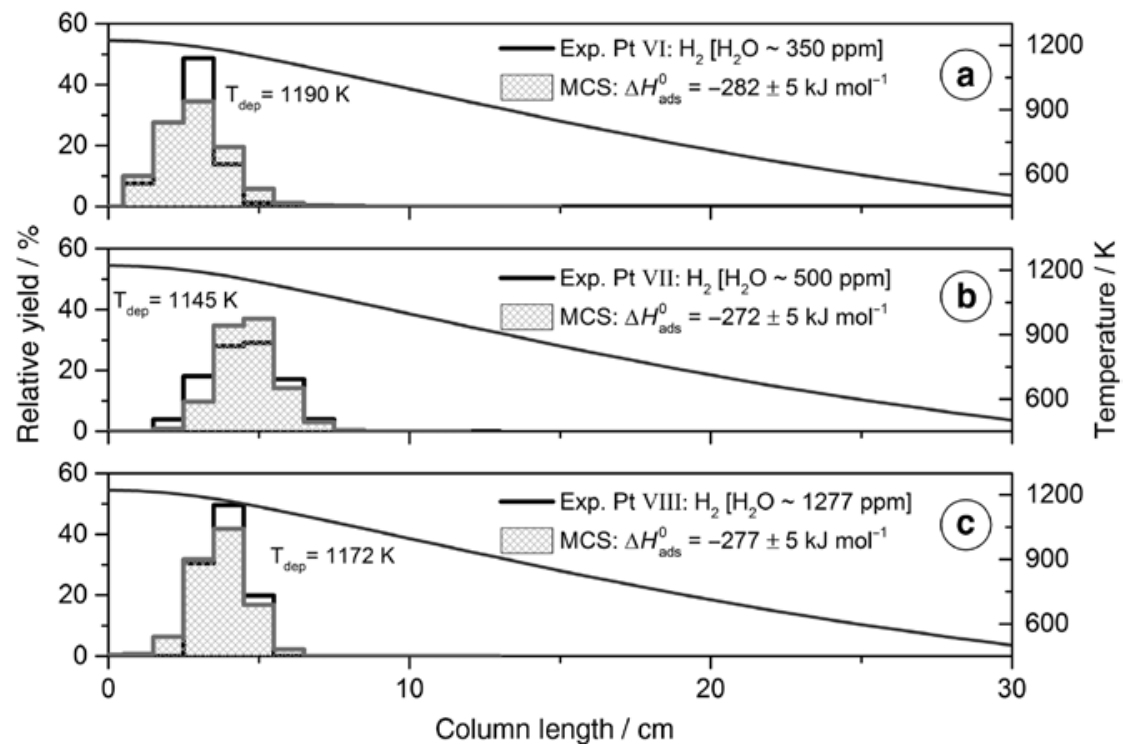

Figure 3: Thermochromatograms of ${ }^{206} \mathrm{Po}$ on platinum using as carrier gas purified $\mathrm{H}_{2}$ with: (a) $350 \mathrm{ppm}$ of $\mathrm{H}_{2} \mathrm{O}$, Exp. Pt VI (black bars), (b) 500 ppm of $\mathrm{H}_{2} \mathrm{O}$, Exp. Pt VII (black bars) and (c) 1277 ppm of $\mathrm{H}_{2} \mathrm{O}$, Exp. Pt VIII (black bars). The light gray cross-hatched bars represent the corresponding Monte Carlo simulations.
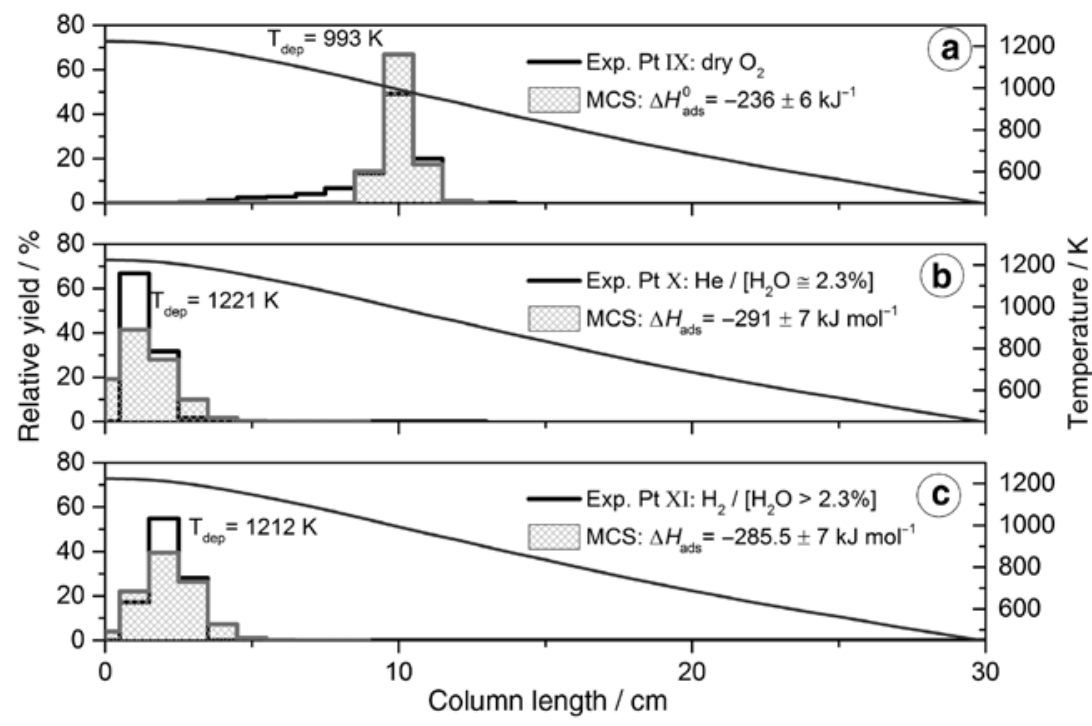

Figure 4: Thermochromatograms of ${ }^{206} \mathrm{Po}$ on platinum. (a) Dry $\mathrm{O}_{2}$ as carrier gas: Exp. Pt IX (black bars). (b) Moist He as carrier gas: Exp. Pt $\mathrm{X}$ (black bars). (c) Moist $\mathrm{H}_{2}$ as carrier gas: Exp. XI (black bars). The light gray cross-hatched bars represent the corresponding Monte Carlo simulations.

reveals a deposition peaking at $993 \mathrm{~K}$ and tailing starting at $1145 \mathrm{~K}$. The adsorption enthalpy of the deposited species was calculated as $-236 \pm 6 \mathrm{~kJ} \mathrm{~mol}^{-1}$, assuming $\mathrm{PoO}_{2}$ as deposited species on a metallic platinum surface.

Exp. Pt X and XI were performed using moist helium and moist $\mathrm{H}_{2}$, respectively, as carrier gases. The two obtained deposition patterns, Figure $4 \mathrm{~b}$ and $\mathrm{c}$, show a main deposition centred at 1221 and $1212 \mathrm{~K}$, respectively. The adsorption enthalpies were calculated as $-291 \pm 7 \mathrm{~kJ}$ $\mathrm{mol}^{-1}$ for experiment Exp. Pt X and $-285.5 \pm 7 \mathrm{~kJ} \mathrm{~mol}^{-1}$ for experiment Exp. Pt XI.

\subsection{Thermochromatography study of polonium on silver}

The interaction between polonium and silver was investigated using purified $\mathrm{He}, \mathrm{H}_{2}$ and dry $\mathrm{O}_{2}$ as carrier gases. 
Additionally, one experiment with moist helium as a carrier gas was performed. The results of the four thermochromatographic experiments are depicted in Figures 5 and 6 and compiled together with the experimental parameters in Table 2.

Figure 5 shows the thermochromatograms obtained using purified $\mathrm{He}$ (Exp. Ag XII) and purified $\mathrm{H}_{2}$ (Exp. Ag XIII), (a) and (b), respectively, as carrier gases. Both thermochromatograms are characterized by a broad deposition pattern, tailing at high temperature and centered at
930 and $990 \mathrm{~K}$, respectively. In both cases a Monte Carlo code [11] was used to estimate the adsorption enthalpy of the deposited species, considering only the portion $(5 \mathrm{~cm})$ of the deposition pattern with the higher relative deposition yield. The Monte Carlo adsorption enthalpy calculations result in $-216 \pm 7$ and $-230 \pm 7 \mathrm{~kJ} \mathrm{~mol}^{-1}$, respectively.

Exp. Ag XIV was conducted in dry $\mathrm{O}_{2}$ atmosphere. The resulting thermochromatogram, Figure $6 \mathrm{a}$, shows a broad deposition pattern ranging from 1171 to $898 \mathrm{~K}$ and peaking at $990 \mathrm{~K}$. Also in this case only the portion of the

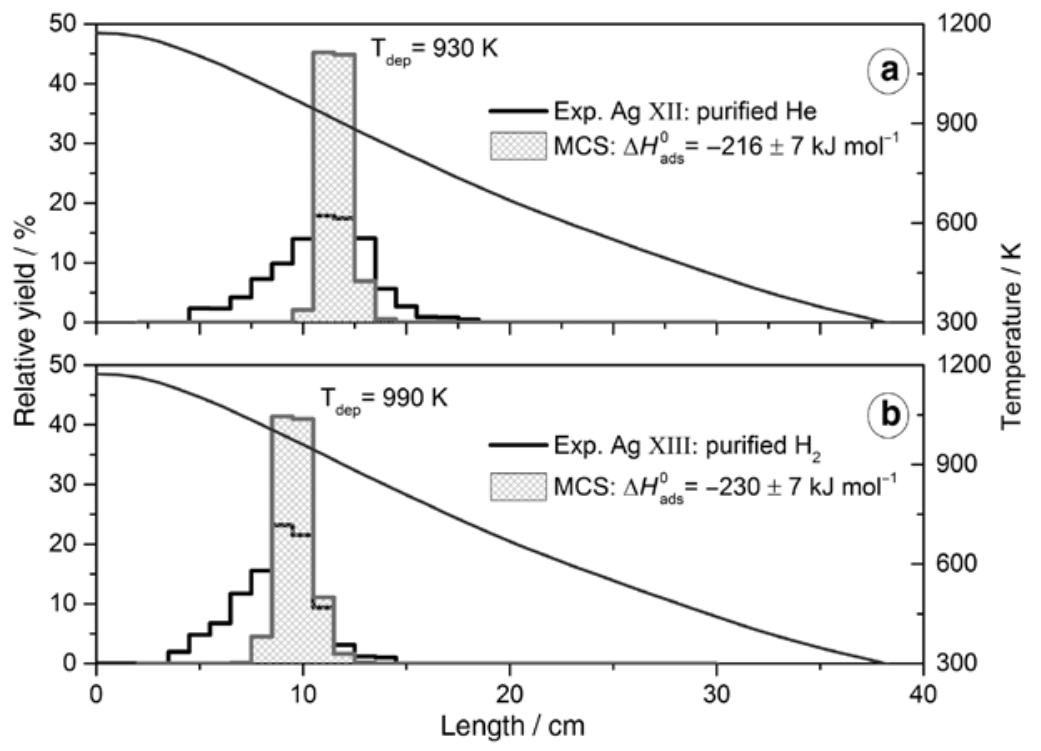

Figure 5: Thermochromatograms of ${ }^{206}$ Po on silver. (a) Purified He as carrier gas: Exp. Ag XII (black bars). (b) Purified $\mathrm{H}_{2}$ as carrier gas: Exp. $\mathrm{Ag}$ XIII (black bars). The light gray cross-hatched bars represent the corresponding Monte Carlo simulations.

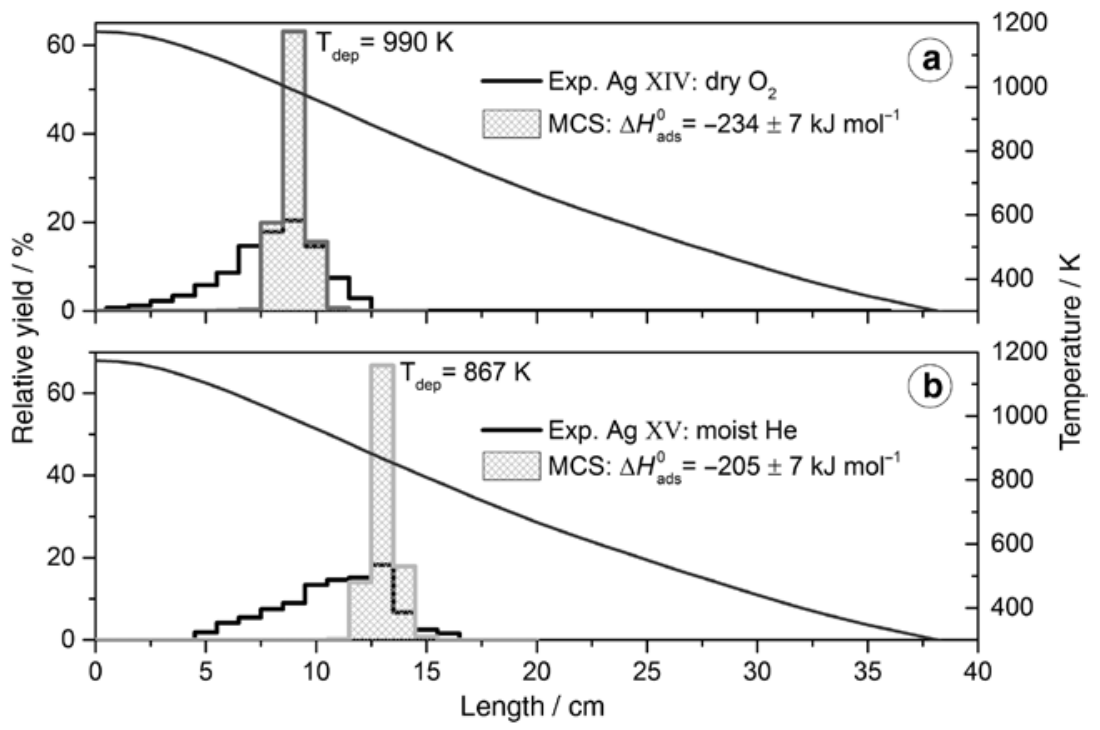

Figure 6: Thermochromatograms of ${ }^{206} \mathrm{Po}$ on silver. (a) Dry $\mathrm{O}_{2}$ as carrier gas: Exp. Ag XIV (black bars). (b) Moist He as carrier gas: Exp. Ag XV (black bars). The light gray cross-hatched bars represent the corresponding Monte Carlo simulations. 
Table 2: Experimental conditions and results of the ${ }^{206}$ Po investigations on silver surfaces.

\begin{tabular}{|c|c|c|c|c|}
\hline $\begin{array}{l}\text { Sample } \\
\text { number }\end{array}$ & Carrier gas & $\begin{array}{r}{ }^{\mathrm{a}} \mathrm{T}_{\text {dep }}{ }^{206} \mathrm{Po} / \mathrm{K} \\
\text { (uncertainty }= \pm 30 \mathrm{~K} \text { ) }\end{array}$ & $\begin{array}{r}\Delta H_{\text {ads }} / \mathrm{kJ} \mathrm{mol}^{-1} \\
\text { (uncertainty }= \pm 7 \mathrm{~kJ} \mathrm{~mol}^{-1} \text { ) }\end{array}$ & Chemical species \\
\hline Exp. Ag XII & Purified He & 930 & -216 & BiPo or Po \\
\hline Exp. Ag XIII & Purified $\mathrm{H}_{2}$ & 990 & -230 & BiPo or Po \\
\hline Exp. Ag XIV & Dry $\mathrm{O}_{2}$ & 990 & -234 & $\mathrm{PoO}_{2}$ \\
\hline Exp. Ag XV & Moist He & 867 & -205 & Po-hydroxides or oxyhydroxides \\
\hline
\end{tabular}

aThe deposition temperature, $\mathrm{T}_{\text {dep }}$, is considered as the temperature corresponding to maximum in the polonium deposition pattern. Considering the uncertainties of temperature and position measurement, its uncertainty is estimated to be $\pm 30 \mathrm{~K}$.

thermochromatogram centered around the maximum of the deposition pattern was used to estimate the adsorption enthalpy of the deposited species by means of the Monte Carlo code [11]. A value of $-234 \pm 7 \mathrm{~kJ} \mathrm{~mol}^{-1}$ was obtained.

Exp. Ag XV was performed using moist helium as a carrier gas. Also in this case the obtained thermochromatogram results in a broad deposition pattern ranging from 1078 to $780 \mathrm{~K}$, peaking at $867 \mathrm{~K}$. The adsorption enthalpy of the main deposited species was calculated, similarly to the Exp. Ag XIV, as $-205 \pm 7 \mathrm{~kJ} \mathrm{~mol}^{-1}$

\subsection{Thermochromatography study of polonium on palladium}

The interaction between polonium and palladium was investigated using purified helium and purified $\mathrm{H}_{2}$ carrier gases. An initial test experiment, using small pieces of palladium foils in oxygen gas was also preformed. The results of the latter showed a severe degradation of the column material due to a strong oxidation of palladium. This indicated the impossibility of studying the interaction of polonium and palladium in oxidizing conditions.

The results obtained in the two experiments performed using palladium lined thermochromatography columns are depicted in Figure 7 and compiled together with the experimental parameters in Table 3. Both thermochromatograms show a sharp deposition pattern centered at $1221 \mathrm{~K}$ for which an adsorption enthalpy of $-340 \pm 7 \mathrm{~kJ}$ mol $^{-1}$ was calculated.

\section{Discussion}

\subsection{Thermochromatography study of polonium on platinum}

The thermochromatogram of the experiment conducted using purified He as carrier gas, Exp. Pt I, is characterized

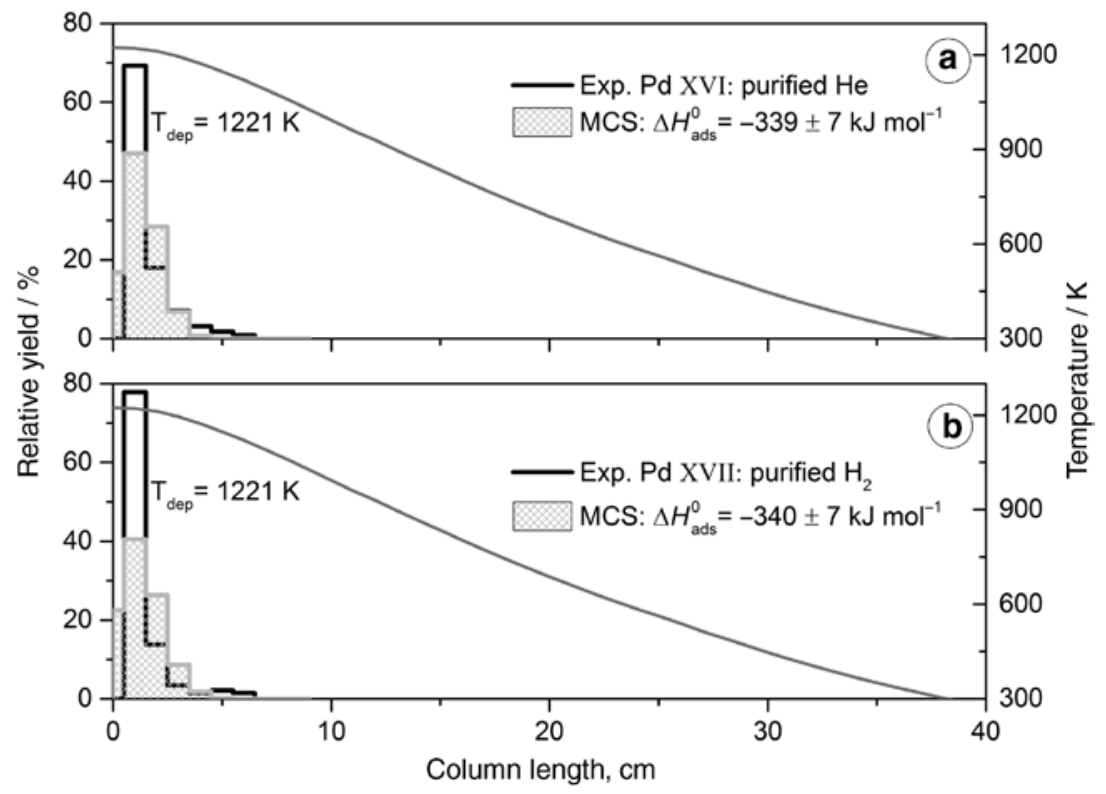

Figure 7: Thermochromatograms of ${ }^{206} \mathrm{Po}$ on palladium. (a) Purified helium as the as carrier gas: Exp. Pd XVI (black bars). (b) Purified $\mathrm{H}_{2}$ as the carrier gas: Exp. Pd XVII (black bars). The light gray cross-hatched bars represent the corresponding Monte Carlo simulations. 
Table 3: Experimental conditions and results of the ${ }^{206} \mathrm{Po}$ investigations on palladium surfaces.

\begin{tabular}{|c|c|c|c|c|}
\hline $\begin{array}{l}\text { Sample } \\
\text { number }\end{array}$ & Carrier gas & $\begin{array}{r}{ }^{\mathrm{a}} \mathrm{T}_{\mathrm{dep}}{ }^{206} \mathrm{Po} / \mathrm{K} \\
\text { (uncertainty }= \pm 30 \mathrm{~K} \text { ) }\end{array}$ & $\begin{array}{r}\Delta H_{\text {ads }} / \mathrm{kJ} \mathrm{mol}^{-1} \\
\text { (uncertainty }= \pm 7 \mathrm{~kJ} \mathrm{~mol}^{-1} \text { ) }\end{array}$ & $\begin{array}{l}\text { Chemical } \\
\text { species }\end{array}$ \\
\hline Exp. Pd XVI & Purified He & 1221 & -340 & $\mathrm{BiPo}$ or $\mathrm{Po}$ \\
\hline Exp. Pd XVII & Purified $\mathrm{H}_{2}$ & 1221 & -340 & $\mathrm{BiPo}$ or $\mathrm{Po}$ \\
\hline
\end{tabular}

${ }^{a}$ The deposition temperature, $T_{\text {dep }}$, is considered as the temperature corresponding to maximum in the polonium deposition pattern. Considering the uncertainties of temperature and position measurement, its uncertainty is estimated to be $\pm 30 \mathrm{~K}$.

by the deposition of one single species at $1026 \mathrm{~K}$, for which an adsorption enthalpy of $-246 \pm 7 \mathrm{~kJ} \mathrm{~mol}^{-1}$ was calculated. On the contrary, in the thermochromatogram resulting from the Exp. Pt II, performed at the same conditions of Exp. Pt I, two peaks are observed, the main one centered at $1058 \mathrm{~K}$, and a small one at $1172 \mathrm{~K}$. The main deposition corresponds to an adsorption enthalpy of $-253 \pm 7 \mathrm{~kJ} \mathrm{~mol}^{-1}$ if no change in the chemical state of the species is assumed. The uncertainties of the calculated adsorption enthalpies are estimated as $\pm 7 \mathrm{~kJ} \mathrm{~mol}^{-1}$. Hence, the species deposited at 1058 and $1026 \mathrm{~K}$ can be considered the same. The presence of unknown amounts of water in the carrier gas or an unstable species initially deposited at higher temperature and not totally decomposed in Exp. Pt II could have led to the observation of polonium traces at higher temperatures, around $1172 \mathrm{~K}$ in the second experiment. Possible candidate species with an expected low volatility and possible high temperature decomposition in the adsorbed state on platinum would be polonium oxyhydroxides or polonium monoxide.

Exp. Pt III-V were conducted using purified $\mathrm{H}_{2}$ as a carrier gas. Although using the same setup and nominally the same conditions, the thermochromatograms resulting from the experiments show different deposition patterns. In particular Exp. Pt III shows a main deposition at $993 \mathrm{~K}$ tailing at high temperature while Exp. Pt IV and V are characterized by a single peak centered at 1090 and $1172 \mathrm{~K}$, respectively. The different results indicate that the used method does not allow for obtaining reproducible conditions when using platinum columns as the stationary phase. This could be due to unknown variation of the water and oxygen content in the carrier gas during the experiments. Their ratio is influenced additionally by catalysis effects induced by the platinum surface at various temperatures.

Another series of three experiments (Exp. VI-VIII) were performed using the same conditions but adding a dew point meter to the outlet of the thermochromatography setup, allowing measuring of the water concentration in the outflowing carrier gas.

Table 1 shows the values of water content measured at the outlet at the end of each experiment. The water content varies from experiment to experiment between 350 and $1277 \mathrm{ppm}$. This clearly indicates that the used gas purification method does not allow for controlling water contents reproducibly. Figure 3 depicts the thermochromatograms of Exp. Pt VI-VIII. It is also observed that in this case the polonium species formed are characterized by different deposition temperature, ranging from 1145 to $1172 \mathrm{~K}$.

The values of the calculated adsorption enthalpies range from -246 to $-282 \mathrm{~kJ} \mathrm{~mol}^{-1}$, indicating a very strong adsorption of all the polonium species deposited on platinum surfaces.

From the reported results (Exp. Pt I-VIII) it is not possible to extract information about the nature of the polonium species formed and deposited on the platinum surface. In purified helium the dominant deposition is observed between 1026 and $1058 \mathrm{~K}$, while in purified hydrogen depositions were observed from 1190 to $993 \mathrm{~K}$ with adsorption enthalpies ranging between -277 and $-256 \mathrm{~kJ} \mathrm{~mol}^{-1}$. In Exp. Pt VI-VIII the measured water content at the outlet of the column did not reveal any systematic correlation between moisture and deposition temperature.

Interestingly, the values of adsorption enthalpies deduced from the experiments are less negative than those predicted for monoatomic polonium on platinum surfaces by DFT calculations [7], while they are in good agreement with the value derived by the MIEDEMA model [8].

A comparison of the adsorption enthalpies calculated in this work with other experimental values reported in literature is difficult due to the large scattering of those values ranging from -187 to $-280 \mathrm{~kJ} \mathrm{~mol}^{-1}[5,17-20]$. The scattering of those literature data and our data emphasizes how experimental conditions, such as differences in the cleanliness and morphologies of the platinum surfaces as well as the purity of the gas phase environment, affect the polonium-platinum adsorption-desorption equilibrium.

The influence of the water contents in the gas phase is more pronounced in Exp. Pt X and XI where moist helium and moist $\mathrm{H}_{2}$ (water concentration approximately $23,000 \mathrm{ppm})$, respectively, are used as carrier gases. The 
obtained thermochromatograms, Figure $4 \mathrm{~b}$ and $\mathrm{c}$, in fact, show the deposition of one single species at 1221 and $1212 \mathrm{~K}$, respectively. This indicates that high concentration of water in the carrier gas induces the formation of one single polonium species, most probably an monoxide or oxyhydroxide [21], with high affinity for platinum, in both $\mathrm{H}_{2}$ and He.

Another sample was studied using dried $\mathrm{O}_{2}$ as carrier gas, Exp. Pt IX. The obtained thermochromatogram (Figure 4a) reveals a deposition pattern peaking at $933 \mathrm{~K}$. In this case the chemical speciation of the deposited species is more certain. In fact, considering the oxidation potential of the carrier gas, the most stable valence state of polonium is +4 [22], thus the species deposited at $993 \mathrm{~K}$ is considered to be $\mathrm{PoO}_{2}$.

The thermochromatogram tails toward high temperature indicating that the resulting species, $\mathrm{PoO}_{2}$, results from a transporting process involving the formation of intermediate species such as polonium oxyhydroxides or polonium monoxide.

Using a Monte Carlo code [11] an adsorption enthalpy of $-236 \mathrm{~kJ} \mathrm{~mol}^{-1}$ was deduced presuming the initial formation and chemical stability of the transported species and metallic platinum as stationary phase. This value indicates that high concentrations of $\mathrm{O}_{2}$ in the cover gas induce the formation of $\mathrm{PoO}_{2}$, which has a lower affinity for platinum compared with all the other polonium species formed in purified and moist $\mathrm{He}$ and $\mathrm{H}_{2}$.

Another important outcome of this study consists in the non-observation of highly volatile polonium species in any of the experiments performed in platinum columns at varied inert and reactive gas compositions. Vice versa, the formation of polonium species volatile even at room temperature was observed in fused silica when using moist helium as the carrier gas [21].

\subsection{Thermochromatography study of polonium on silver and palladium}

The interaction between polonium and silver was investigated using purified He, Exp. Ag XII and purified $\mathrm{H}_{2}$, Exp. Ag XIII, carrier gas.

The results obtained, Figure 5, show deposition patterns peaking at 930 and $990 \mathrm{~K}$, respectively.

The adsorption enthalpy calculated for the Exp. Ag XII and Exp. Ag XIII are -216 and $-230 \mathrm{~kJ} \mathrm{~mol}^{-1}$, respectively.

The values of adsorption enthalpies deduced from the experiments are in good agreement with those predicted for monoatomic polonium on silver surfaces by DFT calculations [7] and by the MIEDEMA model [8].
The difference in deposition temperature between the helium and the hydrogen experiments could be due to the preservation of the BiPo species in the inert gas as suggested [9].

The broadness of the observed depositions can be explained by at least two different processes, which may occur simultaneously. In the first process the species deposited result from a transport reaction process, were one or more intermediate polonium species are formed. The formation of those species, likely polonium hydroxides, could have been enhanced by the presence of trace amounts of water in the carrier gas. Maugeri et al. have hypothesized the formation of polonium hydroxides when performing thermochromatography experiments in fused silica columns using moist helium or moist hydrogen as carrier gases [21].

A second possibility for this observation is the retention of polonium in the adsorbed state due to a superimposed dissolution. This could be expected for metal-metal combination with low absolute values of the partial molar solution enthalpy of the solute (polonium) in the solid phase (metal), $\Delta H_{L S}$. In the case of Ag-Po the $\Delta H_{L S}$ is $4 \mathrm{~kJ} \mathrm{~mol}^{-1}$.

The deposition temperatures and, consequently, the calculated adsorption enthalpies are substantially lower compared to metallic gold [9] and platinum under similar conditions, indicating slightly weaker adsorption.

Exp. Ag XIV was performed using oxidizing carrier gas while Exp. Ag XV was performed using water saturated helium as a carrier.

Both thermochromatograms are characterized by a broad deposition pattern. Similar to the argumentation given above, this could be induced by superimposed transport reactions or by diffusion controlled retention of polonium in the silver bulk.

Using the same argumentation as for the interpretation of the thermochromatography of Exp. Pt IX, we tentatively assign the deposition found at $990 \mathrm{~K}$ to $\mathrm{PoO}_{2}$.

It is interesting to note that in moist helium the deposition of polonium in silver columns is shifted towards lower temperatures compared to purified helium, while in gold [9] and in platinum columns the deposition in moist atmospheres occurs at higher temperatures compared to the drier carrier gases. This could indicate that in presence of moisture and in contact with the silver surfaces polonium compounds, e. g. hydroxides or oxyhydroxides, are indeed slightly more volatile or are bound less strongly to the silver surface than the other observed species.

Similar to the other noble metal surfaces studied so far, also on silver surfaces under all studied conditions, 
polonium is deposited at rather high temperatures, between 867 and $990 \mathrm{~K}$, with adsorption enthalpies ranging from -205 to $-234 \mathrm{~kJ} \mathrm{~mol}^{-1}$, indicating a strong adsorption of the polonium species on the silver surfaces.

Similar to the platinum surfaces, no highly volatile polonium species was observed in any of the experiments performed in silver columns.

It was not possible to perform experiments with palladium in oxidizing carrier gases, as a preliminary test conducted using dry oxygen as a carrier gas with a small foil of palladium, resulted in the complete oxidation of the foil.

Two experiments, performed using palladium lined thermochromatography columns, Exp. Pd XVI and XVII, were conducted using purified helium and $\mathrm{H}_{2}$, respectively. Both resulting thermochromatograms (Figure 7) show the main deposition occurring in the first centimeters of the column, at $1221 \mathrm{~K}$, corresponding to an adsorption enthalpy of $-340 \mathrm{~kJ} \mathrm{~mol}^{-1}$. This indicates that the adsorption interaction of polonium with palladium surfaces is the strongest among the four noble metal surfaces studied here and in Ref. [9], see Table 4.

This agrees reasonably well with predictions of the adsorption enthalpy of single polonium atoms on palladium surfaces obtained by DFT calculations and also with the value predicted by the MIEDEMA model.

This trend is also confirmed by experimental results from the analysis of metal absorbers foils used in the MEGAPIE facility at PSI [23], revealing about two orders of magnitude higher polonium surface concentration on palladium compared to silver.

Comparing the adsorption enthalpies measured in this work (Table 4), there is a noticeable trend that the values obtained when using helium as carrier gas are less negative than the values obtained using hydrogen (with the exception of the adsorption enthalpies of polonium on palladium where the two values are the same). This difference could be due to the different chemical form of the polonium deposited species. In fact, if polonium is present in the starting material as BiPo, this species could be preserved during a thermochromatography experiment when using an inert carrier gas such as helium. While, when using a reactive carrier gas, i.e. hydrogen, BiPo could dissociate in its elements, leading to the deposition of the monoatomic species at higher temperatures.

\section{Conclusions}

All the thermochromatograms of polonium obtained using noble metals as the stationary phase reveal a very strong adsorption of the respective polonium species on the metal surfaces. This supports the idea that these metals can be used as filter materials for the removal of polonium from the gas phase in ADS operated using LBE as coolant and spallation material. In particular, very volatile polonium species formed by the action of water in the carrier gas, as they were observed in experiments in fused silica columns [21] and also in experiments studying the evaporation of polonium from an LBE matrix [24, 25], have not been observed in any of the experiments performed with moist carrier gases in noble metal columns (this work and [9]). As such species might be formed in an ADS reactor by an accidental ingress of water, the ability to safely bind such species is of high importance. In this respect, the present study indicates that such species are either not formed under the conditions prevailing during the experiments, or they are readily decomposed on the noble metal surfaces. Thus, in principle all the noble metals studied here can be considered as viable filter materials. However, it remains to be proven that their capability to supress the formation of volatile polonium species prevails also at the operation temperature of the cover gas system of an ADS.

For answering the question which of the noble metals gold, silver, palladium and platinum is the most suitable candidate for a polonium filter, several aspects have to be

Table 4: Summary of the values of adsorption enthalpy of polonium on palladium, platinum, gold [9] and silver calculated via thermochromatography method, DFT [7] and MIEDEMA model [8].

\begin{tabular}{|c|c|c|c|c|}
\hline \multirow[t]{2}{*}{ Material } & \multicolumn{2}{|c|}{$\Delta H_{\text {ads }}$ determined in this work $/ \mathrm{kJ} \mathrm{mol}^{-1}$} & \multirow{2}{*}{$\begin{array}{r}\Delta H_{\text {ads }} \text { obtained by DFT } \\
\text { calculations } / \mathrm{kJ} \mathrm{mol}^{-1}\end{array}$} & \multirow{2}{*}{$\begin{array}{l}\Delta H_{\text {ads }} \text { calculated by the } \\
\text { MIEDEMA model } / \mathrm{kJ} \mathrm{mol}^{-1}\end{array}$} \\
\hline & Carrier gas He & Carrier gas $\mathrm{H}_{2}$ & & \\
\hline $\mathrm{Pd}$ & -340 & -340 & -298 to -368 & -333 \\
\hline Pt & -246 to -277 & -272 to -282 & -289 to -371 & -260 \\
\hline $\mathrm{Au}^{\mathrm{a}}$ & -207 to -214 & -243 to -249 & -280 & -182 and -274 \\
\hline $\mathrm{Ag}$ & -216 & -230 & -203 to -245 & -219 \\
\hline
\end{tabular}

${ }^{\mathrm{a}} \Delta H_{\text {ads }}$ determined in Ref. [9]. 
considered. The data compiled in Table 4 show that palladium has the highest affinity to polonium among the four metals. However, its tendency to oxidize puts its functionality into question for accident scenarios where ingress of air has to be considered.

Among the remaining metals, platinum has a higher affinity to polonium than gold and silver. However, gold and silver have a less pronounced tendency to form oxides, thus their surfaces may be more stable in accident scenarios with oxygen ingress.

Additionally, factors such as mechanical properties and price may also play a role in the decision for a certain material. Finally, for practical purposes it could prove advantageous to apply high-specific-surface materials such as zeolites impregnated with one of the studied metals instead of the "clean" metal surfaces.

Acknowledgments: This work was supported by the project SEARCH, co-funded by the European Commission under the Seventh Euratom Framework Programme for Nuclear Research \& Training Activities (2007-2011) under Contract Number 295736.

\section{References}

1. Salvatores, M., Palmiotti, G.: Radioactive waste partitioning and transmutation within advanced fuel cycles: achievements and challenges. Prog. Part. Nucl. Phys. 66, 144 (2011).

2. IAEA. Implications of partitioning and transmutation in radioactive waste management. International Atomic Energy Agency, Technical Report Series No. 453. Vienna (2004).

3. Rubbia, C.: A high gain energy amplifier operated with fast neutrons. AIP Conf. Proc. 346, 44 (1995).

4. Sasa, T.: Research activities for accelerator-driven transmutation system at JAERI. Prog. Nucl. Energ. 47, 314 (2005).

5. Eichler, B., Gäggeler, H., Rossbach, H., Hübener, S.: Adsorption of volatile metals on metal surfaces and its application in nuclear chemistry. Radiochim. Acta 38, 131 (1985).

6. Eichler, B., Gaggelerkoch, H., Gaggeler, H.: Thermochromatography of carrier-free elements: polonium in copper columns. Radiochim. Acta 26, 193 (1979).

7. Rijpstra, K.: Density functional theory as a tool to get more out of experimantal data: case-studies for Al-Zn-O and for the interaction between Po and Po-Bi-eutectic. (Ph.D. Thesis), Faculteit Wetenschappen Vakgroep Natuurkunde \& Sterrenkunde, Universiteit Gent, Gent, Belgium (2014).
8. Eichler, B., Rossbach, H.: Adsorption of volatile metals on metalsurfaces and its application in nuclear chemistry. 1. Calculation of adsorption enthalpies for hypothetical superheavy elements with $Z$ around 114. Radiochim. Acta 33, 121 (1983).

9. Maugeri, E. A., Neuhausen, J., Eichler, R., Dressler, R., Rijpstra, K., Cottenier, S., Piguet, D., Vögele, A., Schumann, D.: Adsorption of volatile polonium and bismuth species on metals in various gas atmospheres: Part I - Adsorption of volatile polonium and bismuth on gold. Radiochim. Acta 104, 757 (2016).

10. Zvara, I.: The inorganic radiochemistry of heavy elements. Springer, Netherlands (2010).

11. Zvara, I.: Simulation of thermochromatographic processes by the Monte Carlo method. Radiochim. Acta 38, 95 (1985).

12. Chaston, J. C.: I-the oxidation of platinum. Platinum Metals Rev. 8, 50 (1964).

13. Chaston, J. C.: The oxidation of the platinum metals. A descriptive survey of the reactions involved. Platinum Metals Rev. 19, 135 (1975).

14. Gland, J. L., Sexton, B. A., Fisher, G. B.: Oxygen interactions with the Pt(111) surface. Surf. Sci. 95, 587 (1980).

15. Barin, I.: Thermochemical data of pure substances. Weinheim, New York (1995).

16. Greenwood, N. N., Earnshaw, A.: Chemistry of the elements. Pergaman Press, Oxford (1984).

17. Gäggeler, H., Dornhöfer, H., Schmidt-Ott, W. D., Greulich, N., Eichler, B.: Determination of adsorption enthalpies for polonium on surfaces of copper, silver, gold, palladium and platinum. Radiochim. Acta 38, 103 (1985).

18. Rona, E.: Verdampfungsversuche an polonium. Sitzungsber. Akad. Wiss. Wien 141, 533 (1932).

19. Rona, E., Hoffer, M.: Verdampfungsversuche an Polonium in Sauerstoff und Stickstoff. Sitzungsber. Akad. Wiss. Wien 144, 397 (1935).

20. Hoffer, M.: Über die Bestimmung des Poloniumgehaltes aus Salzen dicker Schichte. Sitzungsber. Akad. Wiss. Wien 144, 393 (1935).

21. Maugeri, E. A., Neuhausen, J., Eichler, R., Piguet, D., Mendonça, T. M., Stora, T., Schumann, D.: Thermochromatography study of volatile polonium species in various gas atmospheres. J. Nucl. Mater. 450, 292 (2014).

22. Abakumov, A. S.: Thermal reactions of polonium. Russ. Chem. Rev. 51, 622 (1982).

23. Hammer-Rotzler, B.: Analysis of the nuclide inventory in MEGA$\mathrm{PIE}$, a proton irradiated lead-bismuth eutectic spallation target. (Ph.D. Thesis), University of Bern, Bern, Switzerland (2015).

24. Rizzi, M., Neuhausen, J., Eichler, R., Tuerler, A., Mendonca, T. M., Stora, T., Prieto, B. G., Aerts, A., Schumann, D.: Polonium evaporation from dilute liquid metal solutions. J. Nucl. Mater. 450, 304 (2014).

25. Gonzáles Prieto, B.: Evaporation of polonium from lead-bismuth eutectic nuclear coolant. (Ph.D. Thesis), KU Leuven, Leuven, Belgium (2015). 